Pacific Journal of Mathematics

MOMENTS OF MEASURES ON CONVEX BODIES 


\section{MOMENTS OF MEASURES ON CONVEX BODIES}

\section{P. H. MASERICK}

In this paper the problem of moments is viewed as one of identifying a class of functions on a semigroup with a class of measures. We present integral representation theorems for linear functionals on algebras (Theorems 2.1 and 2.2) which enable us to solve moment problems for a wide class of compact sets. In particular if $K$ is any compact convex subset of $R^{3}$ with nonvoid interior, necessary and sufficient conditions are given for a triple indexed sequence $f\left(n_{1}, n_{2}, n_{3}\right)$ to admit an integral representation of the form $f\left(n_{1}, n_{2}, n_{3}\right)=\int_{K} t_{1}^{n_{1}} t_{2}^{n_{2}} t_{3}^{n_{3}} d \mu(t)$ $\left(t=\left(t_{1}, t_{2}, t_{3}\right)\right)$. Here, of course the semigroup $S$ considered is all triples of nonnegative integers under coordinate addition. As in the case of Hausdorff's "little moment problems" the solution depends on certain linear combinations of shift operators.

We consider the three coordinate shift operators $E_{1}, E_{2}, E_{3}$ defined on the functions $f$ on $S$, e.g. $\left(E_{2} f\right)\left(n_{1}, n_{2}, n_{3}\right)=f\left(n_{1}, n_{2}+1, n_{3}\right)$. If $K$ is the octahedron $\left\{t \in R^{3} \mid 1 \pm t_{1} \pm t_{2} \pm t_{3} \geqq 0\right\}$. Then $f$ admits a necessarily unique, nonnegative representing measure if and only if $(\Delta f)(0,0,0) \geqq 0$, where $\Delta$ is any product of difference operators of the form $I \pm E_{1} \pm E_{2} \pm$ $E_{3}$. The octahedron example is typical of all bounded nondegenerate convex polyhedra in that the difference operators used to describe those functions which admit representing measures are defined in terms of the iacial functionals of the polyhedron $K$. Necessary and sufficient conditions for the existence of representing signed measures are also given in $\S 3$.

In $\S 4$ we apply Theorems 2.1 and 2.2 to algebras of shift operators on arbitrary commutative semigroups. This leads to a more general notion of functions of Bounded Variation than has previously been introduced on semigroups cf. [6, 11, 12 and 14]. The classical notion of positive definite function on a group is recast in terms of finite difference. We conclude by giving necessary and sufficient conditions for a linear functional on a commutative $B^{*}$-algebra with identity to be in the span of the positive linear functional.

2. Positive and BV-functionals on algebras with involution. Let $\mathscr{A}$ be a real or complex commutative algebra with identity 1 and involution *. If $\mathscr{A}$ is real we assume $x^{*}=x$ for all $x \in \mathscr{A}$. Let $\mathscr{T}$ be a subset of $\mathscr{A}$ such that: 
(i) $\mathscr{T}$ is hermitian $\left(x^{*}=x\right.$ for all $\left.x \in \mathscr{T}\right)$

(ii) $1-\mathscr{T} \subset A \lg \operatorname{span}^{+} \mathscr{T}$ (For each $x \in \mathscr{T}$ there exist products $x_{1}, \cdots, x_{k}$ of members of $\mathscr{T}$ and positive scalars $\alpha_{1}, \cdots, \alpha_{k}$ such that $\left.x+\sum_{t=1}^{k} \alpha_{t} x_{t}=1\right)$

(iii) $\quad$ Alg span $\mathscr{T}=\mathscr{A}$ (For each $x \in \mathscr{A}$ there exist scalars $\alpha_{1}, \cdots, \alpha_{k}$ and products $x_{1}, \cdots, x_{k}$ of members of $\mathscr{T}$ such that $\left.x=\sum_{t=1}^{k} \alpha_{t} x_{t}\right)$. If we set $\operatorname{Alg} \operatorname{span}^{+} \mathscr{T} \equiv P$ then the real span, $\mathscr{A}_{\mathrm{R}}$, of $P$ is $P-P=$ $\{x-y \mid x, y \in P\}$ and the complex span, $\mathscr{A}_{\mathbf{C}}$, of $P$ is $\mathscr{A}_{\mathbf{R}}+i \mathscr{A}_{\mathbf{R}} \equiv$ $\left\{x+i y \mid x, y \in \mathscr{A}_{\mathbf{R}}\right\}$. It follows that $\mathscr{A}_{\mathbf{R}}=\left\{x \in \mathscr{A}_{\mathbf{C}} \mid x=x^{*}\right\}$. Every real, positive homogeneous, additive functional on $P$ admits a unique linear extension to $\mathscr{A}$, this extension necessarily satisfies $f^{*}(x)=f\left(x^{*}\right)$ for all $x \in \mathscr{A}_{\mathrm{C}}$, (we are using * to denote both involution and conjugation). If $f$ is multiplicative on $P$ then so is its extension which we will also denote by $f$. A linear functional $f$ on $\mathscr{A}$ will be called $\mathscr{T}$-positive (or just positive) if $f(x) \geqq 0$ for all $x \in P$. Let $P^{\prime}$ denote the set of all $\mathscr{T}$-positive linear functionals on $\mathscr{A}$. Our main concern is one of characterizing $P^{\prime}$; the real span, $P^{\prime}-P^{\prime}, \quad$ of $P$; and the complex span, $\left(P^{\prime}-P^{\prime}\right)+$ $i\left(P^{\prime}-P^{\prime}\right)$. Throughout we will equip $\operatorname{Span}\left(P^{\prime}\right)$ with the weak * topology.

Proposition 2.1.1. $1-\Pi_{j=1}^{k} x_{j} \in P$ whenever $x_{1}, \cdots, x_{k} \subset \mathscr{T}$.

Proof. The assertion follows from the algebraic identity:

$$
\sum_{\sigma \neq 1} \prod_{j=1}^{k} x_{l}^{\sigma_{l}}\left(1-x_{l}\right)^{1-\sigma_{l}}=1-\prod_{j=1}^{k} x_{l}
$$

where the left summation is taken over all $0-1$ valued functions $\sigma(\cdot)$ defined on the first $k$ natural numbers such that $\sigma \not \equiv 1$. That this identity is valid, follows from induction on $k$ since

$$
\begin{aligned}
& \sum_{\sigma \neq 1} \prod_{j=1}^{k+1} x_{j}^{\sigma_{J}}\left(1-x_{\jmath}\right)^{1-\sigma_{\prime}} \\
& =x_{k+1} \sum_{\sigma \neq 1} \prod_{j=1}^{k} x_{j}^{\sigma_{j}}\left(1-x_{l}\right)^{1-\sigma_{j}} \\
& +\left(1-x_{k+1}\right) \sum_{\sigma \neq 1} \prod_{j=1}^{k} x_{l}^{\sigma_{j}}\left(1-x_{\jmath}\right)^{1-\sigma_{l}}+\prod_{j=1}^{k} x_{j}\left(1-x_{k+1}\right) \\
& =(\text { inductive hypothesis }) \quad\left(1-\prod_{j=1}^{k} x_{\jmath}\right)+\prod_{j=1}^{k} x_{j}\left(1-x_{k+1}\right)=1-\prod_{j=1}^{k+1} x_{j} \text {. }
\end{aligned}
$$

For each $x \in \mathscr{A}$, define the evaluation function $\hat{x}$ on $\operatorname{Span}\left(P^{\prime}\right)$ into the scalar field by $\hat{x}(f)=f(x)$. The above proposition implies $\hat{1}$ is strictly positive on the cone $P^{\prime}$. For if $f \in P^{\prime}$ and $f \not \equiv 0$ then there exist 
$x_{1}, \cdots, x_{k} \in \mathscr{T}$ such that $f\left(\pi_{,} x_{j}\right)>0$ so that $f\left(1-\pi_{j} x_{j}\right) \geqq 0$ implies $\hat{1}(f)=$ $f(1) \geqq f\left(\pi_{j} x_{l}\right)>0$. It follows that $B=\left\{f \in P^{\prime} \mid f(1)=1\right\}$ is a convex base for $P^{\prime}$.

\section{Proposition 2.1.2. The base $B$ is compact.}

Proof. Let $F$ denote the set of all products of members of $\mathscr{T}$ and set $[0,1]_{x}=[0,1]$. Then the restriction $\left.f\right|_{F}$ is a distinct member of the product space $\prod_{x \in F}[0,1]_{x}$ for each $f \in B$. Thus Tychonoff's theorem establishes the assertion since $B^{\prime}=\left\{\left.f\right|_{F}: f \in B\right\}$ is all functions $f$ on $F$ such that $f(1)=1$ and $\sum \alpha_{l} x_{t}=\sum \beta_{l} x_{l}\left(x_{t} \in F\right)$ implies $\sum \alpha_{t} f\left(x_{l}\right)=\sum \beta_{l} f\left(x_{l}\right)$ so that $B^{\prime}$ is closed relative to the topology of simple convergence.

If we let $\Gamma$ denote the class of all $\mathscr{T}$-positive multiplicative functionals on $\mathscr{A}$ then $\Gamma$ is $w^{*}$-compact. Note that $\left.\hat{x} \hat{y}\right|_{\Gamma}=\left.(x y)^{\wedge}\right|_{\Gamma}$ and $\left.\hat{x}\right|_{\Gamma}+\left.\hat{y}\right|_{\Gamma}=\left.(x+y)^{\wedge}\right|_{\Gamma}$.

Theorem 2.1. A linear functional $f$ on $\mathscr{A}$ is $\mathscr{T}$-positive if and only if there exists (a necessarily unique) regular Borel measure $\mu_{f}$ on $\Gamma$ such that

$$
f(x)=\int_{\Gamma} \hat{x}(\chi) d \mu(\chi) .
$$

Proof. The existence of $\mu_{f}$ follows from Choquet's theorem c.f. [4 or 15], once we've shown that the extreme points of $B$ are contained in $\Gamma$. For this we define the translate $f_{x}$ of a linear functional $f$ by $f_{x}(y)=f(x y)(x, y \in \mathscr{A})$ then $f_{x}$ is linear and $\mathscr{T}$-positive if $f$ is $\mathscr{T}$-positive and $x \in P$. Since $f=f_{x}+f_{1-x}$ for $x \in \mathscr{T}$ we have $\lambda f=f_{x}$ whenever $f$ is an extreme point. Evaluation at 1 shows $\lambda=f(x)$ so that $f(x) f(y)=$ $f(x y)$ for all $y \in \mathscr{A}$ and $x \in \mathscr{T}$. It follows that $f$ is multiplicative so that every extreme point of $B$ is multiplicative. See [1] for a similar argument. Uniqueness follows from the Stone-Weierstrass theorem since $\left\{\left.\hat{x}\right|_{\mathrm{r}}: x \in \mathscr{A}\right\}$ is a point separating ${ }^{*}$-subalgebra of the continuous functions $\mathscr{C}(\Gamma)$ on $\Gamma$ which contains constants.

The argument used in [5] shows that every member of $\Gamma$ is in fact extreme so that $B$ is a Bauer Simplex, i.e. a simplex whose set of extreme points is closed. Since the map $f \rightarrow \mu_{f}$ of $P$ onto the nonnegative measures is linear it is natural to ask what linear functionals admit a representing measure with respect to real and complex measures? I.e., how can $P^{\prime}-P^{\prime}$ and $\left(P^{\prime}-P^{\prime}\right)+i\left(P^{\prime}-P^{\prime}\right)$ be characterized? The answer to this question relies on the concept of $B V$-function and the proofs which we will use depend heavily on compactness arguments with certain partitions of unity. We begin with some preliminary definitions.

A cycle is a finite subset $\left\{x_{1}, \cdots, x_{k}\right\}$ of $P$ such that $x_{1}, \cdots, x_{k-1} \in \mathscr{T}$ and $\sum_{\imath=1}^{k} x_{l}=1$. Let $\mathscr{P}$ be a collection of cycles such that each $x \in \mathscr{T}$ is 
in at least one cycle of $\mathscr{P}$. An example of such a $\mathscr{P}$ is $\{x, 1-x \mid x \in$ $\mathscr{T}\}$. The cycle $\{x, 1-x\}$ will be called a simple cycle. The motivation for considering simple cycles comes from considering the algebra $\mathscr{A}$ of shift operators on the additive semigroup $S$ of nonnegative integers in light of Hausdorff's solution to the "little moment problem" [cf. 16]. These cycles correspond to the collection $\left\{\left\{t^{k}(1-t)^{n-k}\right\}_{k \leqq n}\right\}_{n}$ of partitions of unity on $\Gamma \equiv[0,1]$ and this collection is rich enough to adequately describe the variation of the Borel measures on $\Gamma$. When dealing with more general semigroups we must consider products of these cycles to obtain rich enough partitions. In this generality $\Gamma=$ $\exp (S)$ [cf. 11]. In trying to describe the variation of measures supported by compact subsets of the semicharacters of $S$ other than $\exp S$, it becomes necessary to consider other than simple cycles. This becomes particularly important for many of the applications given later and we feel justifies the additional complexity. With each finite subcollection $\left\{\left\{x_{1}\right\}_{1}\right\}_{\text {, }}$ of $\mathscr{P}$, we associate the partition of unity of all distinct products which contain one factor from each cycle. When the cycles in the subcollection agree for each $j=1,2, \cdots, n$ we get the multinomial partition

$$
\left\{\left(\begin{array}{c}
n \\
i_{1}, \cdots, i_{m}
\end{array}\right)\left(x_{1}^{i_{1}} \cdots x_{m}^{i_{m}}\right) \mid \sum_{l=1}^{m} i_{l}=n\right\} .
$$

In general we will be considering products of multinomial partitions of cycles in $\mathscr{P}$, i.e. partitions of the form

$$
\left\{\left(\begin{array}{c}
n_{1} \\
i_{11}, \cdots
\end{array}\right) \cdots\left(\begin{array}{c}
n_{k} \\
i_{1 k}, \cdots
\end{array}\right) \prod_{l, j} x_{l^{\prime \prime}}^{i_{l}} \mid \sum_{l} i_{l j}=n_{l}\right\}
$$

where $\left\{x_{l\}}\right\}_{l}$ is a cycle for each $j=1, \cdots, k$. If we set the expression under the bracket equal to $\boldsymbol{p}$ then the functions $\hat{\boldsymbol{p}}$ (restricted to $\Gamma$ ) form a partition of unity in the space of continuous functions $\mathscr{C}(\Gamma)$. We will consider these products of multinomial partitions of unity in $\mathscr{C}(\Gamma)$ extensively later and without further reference. For each finite subset $\mathscr{P}_{0}=\left\{\left\{x_{i j}\right\}\right\}_{1=1,2, \cdots, k}$ of $\mathscr{P}$ and each set of integers $\left\{n_{1}, \cdots, n_{k}\right\}$ define

$$
\|f\|_{\left(\mathscr{P}_{0, n}, n_{1}\right)}=\sum\left(\begin{array}{c}
n_{1} \\
i_{11}, \cdots
\end{array}\right) \cdots\left(\begin{array}{c}
n_{k} \\
i_{1 k}, \cdots
\end{array}\right)\left|f\left(\prod_{l, j} x_{l, j}^{i_{l, l}}\right)\right|,
$$

where summation is taken with respect to all choices of $i_{l,}$ such that $\Sigma_{l} i_{l j}=n$, for each $j=1,2, \cdots, k$.

Note that if $\mathscr{P}_{0}$ is a collection of simple cycles $\left\{x_{j}, 1-x_{j}\right\}_{j=1,2, k, k}$ then

$$
\|f\|_{\mathscr{P}_{0}}=\sum\left(\begin{array}{c}
n_{1} \\
i_{1}
\end{array}\right) \cdots\left(\begin{array}{c}
n_{k} \\
i_{k}
\end{array}\right)\left|f\left[\Pi_{l} x_{j}^{i_{j}}\left(1-x_{l}\right)^{n_{j}-i_{j}}\right]\right|
$$


Finally we define the total variation of $f$, by

$$
\|f\|=\sup _{\left(\mathfrak{P}_{1}, n_{l}\right)}\|f\|_{\left(\mathscr{P}_{1}, n_{3}\right)}
$$

and $\left(\mathscr{P}_{0}, n_{l}\right) \leqq\left(\mathscr{P}_{0}^{\prime}, n_{j}^{\prime}\right)$ if $\mathscr{P}_{0} \subset \mathscr{P}_{0}^{\prime}$ and $n_{l} \leqq n^{\prime}$, for corresponding $j$ 's. The class of all functionals of finite variation are called $\mathscr{T}-B V$ (or just BV) and it is this class of functionals which we will show admit integral representations. Our main theorem is as follows.

THEOREM 2.2. The BV-functionals form the linear span of the positive linear functionals. These are precisely the linear functionals $f$ which admit a (necessarily unique) representing measure $\mu_{f}$. If $f$ is $\mathrm{BV}$ then $\left\|\mu_{f}\right\|=\|f\|$ and $\mu_{f}$ is real if and only if $f\left(x^{*}\right)=f^{*}(x)$.

The proof is broken down into a series of lemmas. Lemmas 2.2.1 through 2.2.3 prove that if $f$ admits a representing measure then $f$ is $\mathrm{BV}$.

LEMMA 2.2.1. Let $\varepsilon>0$ be given and $K_{1}$ and $K_{2}$ be disjoint compact subsets of $\Gamma$. There exists a product $\Lambda$ of multinomial partition of cycles in $\mathscr{P}$ with subpartition $\Lambda_{0}$ such that

$$
\sum_{p \in \Lambda_{0}}\left|\int \hat{p} d u\right| \begin{cases}\geqq 1-\epsilon & \text { on } K_{1} \\ <\epsilon & \text { on } K_{2} .\end{cases}
$$

Proof. Let $\chi_{1}$ and $\chi_{2}$ be distinct members of $\Gamma$. Then there exists $x_{1} \in \mathscr{T}$ such that $\chi_{1}\left(x_{1}\right) \neq \chi_{2}\left(x_{1}\right)$. These two real numbers can be separated by disjoint open subsets $G_{1}$ and $G_{2}$ in $[0,1]$ with $\chi_{\lrcorner}\left(x_{1}\right) \in G_{j}$ for $j=1,2$. The theory of Bernstein polynomials cf. [8, page 6] implies that for all sufficiently large $n$ the partition of unity $\left\{\left(\begin{array}{c}n \\ i\end{array}\right) t^{i}(1-t)^{n-1}\right\}$ $(0 \leqq t \leqq 1)$ admits a subpartition which is arbitrarily close to zero on $G_{2}$ and arbitrarily close to unity on $G_{1}$. Inverting, we have the existence of a subpartition of the partition of unity $\left\{\left(\begin{array}{c}n \\ i\end{array}\right) \hat{x}_{1}^{\prime}\left(1-\hat{x}_{1}\right)^{n-i}\right\}$ which is arbitrarily close to zero on the preimage $\hat{x}_{1}^{\leftarrow}\left(G_{2}\right)$ and arbitrarily close to unity on $\hat{x}_{1}^{\leftarrow}\left(G_{1}\right)$. But if $\left\{x_{i}\right\}_{i=1,2, \cdots, k}$ is a cycle in $\mathscr{P}$ which contains $x_{1}$, then

$$
\begin{aligned}
& \sum\left(\begin{array}{c}
n \\
i_{1}, i_{2}, \cdots, i_{k}
\end{array}\right) x_{1}^{i_{1}} \cdots x_{k}^{i_{k}} \\
& \quad=\sum_{i_{1}=0}^{n}\left(\begin{array}{c}
n \\
i_{1}
\end{array}\right) x_{1}^{i_{1}} \sum\left(\begin{array}{c}
n-i_{1} \\
i_{2} \cdots i_{k}
\end{array}\right) x_{2}^{i_{2}} \cdots x_{k}^{i_{k}}=\sum_{i=0}^{n}\left(\begin{array}{c}
n \\
i
\end{array}\right) x_{1}^{i}\left(1-x_{1}\right)^{n-1} .
\end{aligned}
$$

Thus the partition of unity 


$$
\left(\begin{array}{c}
n \\
i_{1}, \cdots, i_{k}
\end{array}\right) \hat{x}_{1}^{t_{1}} \cdots \hat{x}_{k}^{i_{k}}
$$

admits a subpartition which separates the disjoint open sets $\hat{x}_{\uparrow}^{\leftarrow}\left(G_{1}\right)$ and $\hat{x}_{1}^{\leftarrow}\left(G_{2}\right)$ which contain the singleton (compact) sets $\left\{\chi_{1}\right\}$ and $\left\{\chi_{2}\right\}$ as in Lemma 3.1 of [10]. The compactness argument used there implies the present lemma.

LEMMA 2.2.2. Let $\mu$ be a bounded complex valued measure, $f$ and $g$ integrable functions and $E$ a measurable set. If $1 \geqq f \geqq 1-\epsilon$ and $0 \leqq g \leqq$ $\epsilon$ on $E$ then

$$
\left|\int_{E} f d u\right| \geqq(1-4 \epsilon)|\mu(E)| \text { and (ii) }\left|\int_{E} g d u\right| \leqq 4 \epsilon|\mu(E)| \text {. }
$$

Proof.

$$
\text { (i) } \begin{aligned}
|\mu(E)| & -\left|\int_{E} f d u\right| \leqq\left|\mu(E)-\int_{E} f d u\right| \\
& \leqq \int_{E}(1-f) d|\mu| \leqq \epsilon|\mu|(E) \leqq 4 \epsilon|\mu(E)|
\end{aligned}
$$

Similarly (ii) $\left|\int_{E} g d u\right| \leqq \int_{E} g d \mu\left|\leqq \int_{E} \epsilon d\right| \mu|\leqq 4 \epsilon| \mu(E) \mid$.

LEMMA 2.2.3. If $\mu$ is a complex regular Borel measure on $\Gamma$ then the variation is given by

$$
\|\mu\|=\sup _{\Lambda} \sum_{p \in \Lambda}\left|\int \hat{p} d \mu\right|
$$

where $\Lambda$ is an arbitrary product of multinomial partitions of cycles in $\mathscr{P}$.

Proof. Choose $\epsilon$ such that $0<\epsilon<1$. From regularity, there exists disjoint compact sets $\left\{K_{i} \mid i=1,2, \cdots, k\right\}$ such that

(i) $\|\mu\|-\epsilon / 3 \leqq \sum_{i=1}^{k}\left|\mu\left(K_{i}\right)\right|$.

From Lemma 2.2.1, there exists a collection $\left\{\Lambda^{\prime}\right\}_{\text {, }}$ of products of multinomial partitions with subpartitions $\left\{\Lambda_{0}^{\prime}\right\}_{\text {, such }}$ that

$$
\text { (ii) } \sum_{p \in \Lambda_{0}^{\prime}} \hat{p}(x) \begin{cases}\geqq \sqrt[k]{1-\epsilon / 12} & \text { on } K_{t} \\ <1-\sqrt[k]{1-\epsilon / 12 k} & \text { on } \bigcup_{j \neq i} K_{,} .\end{cases}
$$


Let $\Lambda=\Pi_{1} \Lambda^{\prime}=\left\{\boldsymbol{p}_{1}, \boldsymbol{p}_{2} \cdots \boldsymbol{p}_{k} \mid \boldsymbol{p}_{t} \in \Lambda^{i}\right\}$. Then $\Lambda$ is a product of multinomial partitions with subpartitions

$$
\Lambda_{\infty}^{\prime}=\Lambda_{0}^{t} \prod_{\mid \neq 1}\left(\Lambda^{\prime} \backslash \Lambda_{0}^{\prime}\right)
$$

Since $\sqrt[k]{1-\epsilon / 12 k} \geqq \sqrt[k]{1-\epsilon / 12}$, Lemma 2.2 .2 implies

$$
\left|\int_{K_{1}} \sum_{p \in \Lambda_{\propto}^{\prime}} \hat{p} d \mu\right| \geqq\left(1-\frac{\epsilon}{3}\right)\left|\mu\left(K_{\imath}\right)\right| \text {. }
$$

Similarly, since $1-\sqrt[k]{1-\epsilon / 12 k}<\epsilon / 12 k$ we have

$$
\left|\int_{\substack{\cup K_{i} \\ j \neq l^{\prime}}} \sum_{p \in \Lambda_{\dot{x}}^{\prime}} p d \mu\right| \leqq \frac{\epsilon}{3 k}\left|\mu\left(\bigcup_{j \neq i} K_{j}\right)\right|<\frac{\epsilon}{3 k}\|\mu\| .
$$

Therefore

$$
\begin{aligned}
& \sum_{p \in \Lambda}\left|\int \hat{p} d \mu\right| \geqq \sum_{1} \sum_{p \in \Lambda_{x}^{\prime}}\left|\int \hat{p} d \mu\right| \\
& \geqq \sum_{i} \sum_{p \in \Lambda_{\dot{x}}^{\prime}}\left[\left|\int_{K_{i}} \hat{\boldsymbol{p}} d \mu\right|-\left|\int_{\substack{\cup K_{I} \\
j \neq i}} \boldsymbol{p} d \mu\right|-\left|\int_{\substack{\Gamma \mid \cup K_{i} \\
j \neq i}} \hat{\boldsymbol{p}} d \mu\right|\right] \\
& \geqq \sum_{i}\left(1-\frac{\epsilon}{3}\right)\left|\mu\left(K_{\imath}\right)\right|-\sum_{i} \frac{\epsilon}{3 k}\|\mu\|-\sum_{p \in \Lambda}\left|\int \hat{p} d \mu\right| \\
& \geqq\left(1-\frac{\epsilon}{3}\right) \sum\left|\mu\left(K_{\imath}\right)\right|-\frac{\epsilon}{3}\|\mu\|-\sum_{p \in \Lambda} \int \hat{p} d|\mu| \\
& \geqq \text { (by i) }\left(1-\frac{\epsilon}{3}\right)\left[\|\mu\|-\frac{\epsilon}{3}\right]-\frac{\epsilon}{3}\|\mu\|-|\mu|\left(\Gamma \backslash \cup K_{\jmath}\right) \text {. }
\end{aligned}
$$

Since $|\mu|(\Gamma)-\epsilon / 3 \leqq \Sigma|\mu|\left(K_{\imath}\right)$, we have $|\mu|\left(\Gamma \mid \cup K_{l}\right)<\epsilon / 3$ so that

$$
\sum_{p \in \Lambda}\left|\int p d \mu\right| \geqq\left(1-\frac{2}{3} \epsilon\right)\|\mu\|-\frac{2}{3} \epsilon \text { or }\|\mu\| \leqq \sup _{\Lambda} \sum_{\Lambda}\left|\int \hat{p} d \mu\right| \text {. }
$$

The assertion follows since

$$
\sum_{\wedge}\left|\int \hat{p} d \mu\right| \leqq \sum_{\wedge} \int \hat{p} d|\mu|=\|\mu\| .
$$

REMARK 2.2.4. If $f$ admits a representing measure $\mu_{f}$ then $\left\|\mu_{f}\right\|$ $=\|f\|$. 


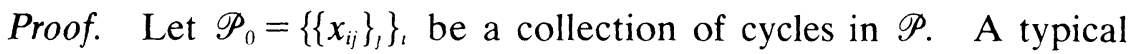
member of a product $\Lambda$ of multinomial partitions of $\mathscr{P}$ of the form:

$$
\boldsymbol{p}=\left(\begin{array}{c}
n_{1} \\
i_{11}, \cdots
\end{array}\right) \cdots\left(\begin{array}{c}
n_{k} \\
i_{1 k}, \cdots
\end{array}\right) \Pi_{l, j} x_{l_{l, j}^{\prime \prime}}
$$

so that

$$
\|f\|_{\left(\mathcal{P}_{0}, n_{1}\right)}=\sum_{p \in \Lambda}|f(\boldsymbol{p})|=\sum_{p \in \Lambda}\left|\int \hat{p} d \mu_{f}\right|
$$

Lemma 2.2.3 implies the remark.

In order to prove that every BV-functional admits a representing measure we will define the variation of a BV-functional $f$ by $|f|(y)=$ $\left\|f_{y}\right\|$ for all $y \in P$. If $f \in P^{\prime}-P^{\prime}$ then $\mu_{f_{y}}=\hat{y} d \mu_{f}$ since $f_{y}(x)=f(y x)=$ $\int y x d \mu_{f}$. Hence $|f|(y)=\left\|f_{y}\right\|=\left\|\mu_{f_{y}}\right\|=\left\|\hat{y} d \mu_{f}\right\|$ and since $\hat{y}$ is nonnegative on $\Gamma,\left\|\hat{y} d \mu_{f}\right\|=\int \hat{y} d\left|\mu_{f}\right|$. Therefore $|f|(y)=\int \hat{y} d\left|\mu_{f}\right|$ for all $y \in P$. Once we show $|f|(\cdot)$ is finite, additive and positive homogeneous on $P$ we can linearly extend $|f|$ to $\mathscr{A}_{\mathbf{R}}$ whenever $f$ is real on $\mathscr{A}_{\mathbf{R}}$. It will be shown that $f=1 / 2(|f| \pm f)$ is $\mathscr{T}$-positive so that $f=$ $f^{+}-f^{-} \in P^{\prime}-P^{\prime}$ whenever $f$ is real on $\mathscr{A}_{\mathbf{R}}$ and BV. The complex case easily follows. We will need to verify some preliminary properties of the total variation first. We begin with the following elementary consequence of the triangle inequality and positive homogeneity of the absolute value.

LEMMA 2.2.5.

(i) $\|f+g\|_{\mathscr{P}_{0}} \leqq\|f\|_{\mathscr{P}_{0}}+\|g\|_{\mathscr{P}_{0}}$

(ii) $\|\alpha f\|_{\mathcal{P}_{1}}=|\alpha| \cdot\|f\|_{\mathscr{P}_{1}}$

Thus $\|\cdot\|$ is a positive, homogeneous, subadditive function on the $\mathrm{BV}$-linear functionals.

Let $\left\{\left\{x_{l j}\right\}_{l}\right\}_{\text {, }}$, be a finite subset of cycles. Then since $\left(x_{11}+\cdots\right)^{n_{1}}\left(x_{12}+\right.$ $\cdots)^{n_{2}} \cdots\left(x_{1 l}+\cdots\right)^{n_{1}+1} \cdots\left(x_{l k}+\cdots\right)^{n_{k}}=$ $\left(x_{11}+\cdots\right)^{n_{1}}\left(x_{12}+\cdots\right)^{n_{2}}\left(x_{1 l}+\cdots\right)^{n_{l}} \cdots\left(x_{1 k}+\cdots\right)^{n_{k}}\left(x_{1 l}+\cdots\right)$,

$$
\|f\|_{\left(\mathscr{P}_{0} ; n_{1}, \cdots, n_{l+1}, \cdots, n_{k}\right)}=\|f\|_{\left(\mathscr{P}_{1} ; n_{1}, \cdots, n_{k}, 1\right)}
$$

where $\mathscr{P}_{1}$ is the union of $\mathscr{P}_{0}$ with the single cycle $\left\{x_{1 l}, x_{21}, \cdots\right\}$. By induction we can eliminate all repetitious cycles by increasing the order. In summary we present the following.

LeMMA 2.2.6. Let $\mathscr{P}_{0}$ be a finite collection of cycles and $\mathscr{P}_{0}^{\prime}$ be the 
collection of all pairwise distinct cycles of $\mathscr{P}_{0} . \quad$ Then $m_{1}, m_{2}, \cdots, m_{l}$ can be chosen so that

$$
\|f\|_{\left(\mathscr{P}_{0} ; n_{1}, \cdots, n_{k}\right)}=\|f\|_{\left(\mathscr{P}^{\prime}, m_{1}, \cdot, m_{l}\right)} .
$$

LEMMA 2.2.7. Let $\mathscr{P}_{1}=\left\{x_{1}, x_{2,}, \cdots\right\}_{j=1, \cdots, m}$ be a set of $m$ cycles and let $\mathscr{P}_{0}$ be the first $k-1$ of them. Then

$$
\|f\|_{\left(\mathscr{P}_{\left.1, n_{j}\right)}\right.}=\sum\left(\begin{array}{c}
n_{k} \\
i_{1 k}, \cdots
\end{array}\right) \cdots\left(\begin{array}{c}
n_{m} \\
i_{1 m}, \cdots
\end{array}\right)\left\|f_{x}\right\|_{\left(\mathscr{P}_{0,}, n_{j}\right)} \text { where } x=\prod_{j=k}^{m}\left(x_{1, j}^{i_{1}, \cdots}\right) .
$$

Proof.

$\|f\|_{\left(\mathscr{P}_{1, n}, n_{1}\right)}=\sum\left(\begin{array}{c}n_{k} \\ i_{1 k}, \cdots\end{array}\right) \cdots\left(\begin{array}{c}n_{m} \\ i_{1 m}, \cdots\end{array}\right) \sum\left(\begin{array}{c}n_{1} \\ i_{11} \ldots\end{array}\right) \cdots\left(\begin{array}{c}n_{k-1} \\ i_{1 k+1} \ldots\end{array}\right)\left|f_{x}\left(\prod_{j=1}^{k} x_{1 j}^{i_{1 j}} \ldots\right)\right|$

and the lemma follows.

If we take $m=k$ and $n_{m}=1$ then the above lemma along with Lemma 2.2.5 shows $\|f\|_{\left(\mathscr{P}_{1, n}, n_{j}\right)}=\Sigma_{l}\left\|f_{x k l}\right\|_{\left(\mathscr{P}_{1}, n_{1}\right)} \geqq\left\|\Sigma_{l} f_{x k l}\right\|=\|f\|_{\left(\mathscr{P}_{1}, n_{l}\right)}$. Lemma 2.2.6 now implies the following.

LEMMA 2.2.8. $\|f\|_{\left(\mathscr{P}_{0, n}, n_{1}\right.}$ is a nondecreasing function of $\mathscr{P}_{0}$ and $n_{j}$. In particular

$$
\|f\|=\lim _{\left(\mathscr{P}_{0}, n_{j}\right)}\|f\|_{\left(\mathscr{P}_{0}, n_{1}\right)} .
$$

LEMMA 2.2.9. If $x$ is a finite product of member of $\mathscr{T}$ and $f$ is BV then $f_{x}$ and $f_{1-x}$ are $\mathrm{BV}$ and $\|f\|=\left\|f_{x}\right\|+\left\|f_{1-x}\right\|$.

Proof. Let $x=\prod_{j=1}^{k} x_{1,}\left(x_{1 j} \in \mathscr{T}\right)$ and $\mathscr{P}_{0}$ be a finite subset of $\mathscr{P}$. For each $j$, there exists a cycle $C_{j}=\left\{x_{1 j}, x_{2,}, \cdots, x_{l_{j j}}\right\}$ contained in $\mathscr{P}$ which contains $x_{1}$. Let $\mathscr{P}_{1}$ be the collection of cycles obtained by adjoining $C_{k}$, with multiplicity 1 , to $\mathscr{P}_{0}$ for each $j$. Then from Lemma 2.2.7,

$$
\|f\|_{\mathscr{P}_{1}}=\sum_{\eta(\cdot)}\left\|f_{y_{\eta}}\right\|_{\mathscr{P}_{0}}
$$

where $\eta(j) \in\left\{1,2, \cdots, l_{j}\right\}$ and $y_{\eta}=\Pi_{j} x_{\eta()) j}$. Hence

$$
\|f\| \geqq \sum_{\eta}\left\|f_{y_{\eta}}\right\|_{\mathcal{B}_{0}} \geqq\left\|f_{x}\right\|_{\mathscr{P}_{0}}+\sum_{\eta \neq 1}\left\|f_{y_{\eta}}\right\|_{\mathscr{P}_{0}} \rightarrow\left\|f_{x}\right\|+\left\|f_{1-x}\right\| \geqq\|f\|
$$


The assertion follows.

Lemma 2.2.10. $|f|(\cdot)$ is additive and positive homogeneous on $P$.

Proof. Let $x=\sum \alpha_{1} x_{i}\left(\alpha_{1} \geqq 0, x_{i} \in \mathscr{T}^{k}\right)$ be an arbitrary member of P. Then

$$
\begin{aligned}
\left\|\sum_{i} \alpha_{\imath} f\right\| & =\left\|\sum_{i} \alpha_{i}\left(f_{x_{i}}+f_{1-x_{i}}\right)\right\| \leqq\left\|\sum_{i} \alpha_{i} f_{x_{i}}\right\|+\left\|\sum_{i} \alpha_{i} f_{1-x_{i}}\right\| \\
& \leqq \sum \alpha_{\imath}\left\|f_{x_{i}}\right\|+\sum \alpha_{i}\left\|f_{1-x_{i}}\right\|=\sum_{i} \alpha_{i}\|f\|=\left\|\sum_{i} \alpha_{t} f\right\| .
\end{aligned}
$$

A second application of the triangle inequality shows $\left\|\sum \alpha_{i} f_{x_{i}}\right\|=\sum \alpha_{i}\left\|f_{x_{i}}\right\|$ so that

$$
|f|\left(\sum \alpha_{i} x_{i}\right)=\left\|f_{\sum \alpha_{x_{i}}}\right\|=\left\|\sum \alpha_{i} f_{x_{i}}\right\|=\sum \alpha_{i}\left\|f_{x_{i}}\right\|=\sum \alpha_{i}|f|\left(x_{i}\right) .
$$

LEMMA 2.2.11. If $f$ is a linear BV-functional on s such that $f^{*}(x)=f\left(x^{*}\right)$ for all $x \in \mathscr{A}$ then $f$ is the difference of two positive functionals.

Proof. Note that $f$ is real on $P$. Since $|g(1)| \leqq\|g\|$ for all $g$, we have $|f(x)|=\left|f_{x}(1)\right| \leqq\left\|f_{x}\right\|=|f|(x)$ so that $|f|(x) \geqq \pm f(x)$ is for all $x \in P$. Thus $f^{ \pm}=1 / 2[|f| \pm f]$ is $\mathscr{T}$-positive whenever $f$ is BV. The assertion follows since $f=f^{+}-f^{-}$.

Theorem 2.1 now implies that a BV-functional $f$ admits a real representing measure if and only if $f^{*}(x)=f\left(x^{*}\right)$ for all $x \in \mathscr{A}$. The proof of Theorem 2.2 will be complete once we've established the following lemma.

Lemma 2.2.12. If $\mathscr{A}$ is complex then the set $\mathrm{BV}(\mathscr{A})$ of $\mathrm{BV}$ functionals is $\left(P^{\prime}-P^{\prime}\right)+i\left(P^{\prime}-P^{\prime}\right)$.

Proof. For each BV-functional $f$, set $f_{*}(x)=f^{*}\left(x^{*}\right)$. Then $f_{*}$ is linear. Also $f=f_{1}+i f_{2}$, where $f_{1}=\left(f+f_{*}\right) / 2$ and $f_{2}=\left(f-f_{*}\right) / 2 i$. Since $x=x^{*}$ for all $x \in \mathscr{T}$ we have $\left|f_{*}\left(\Pi x_{j}\right)\right|=\left|f\left(\Pi x_{j}\right)\right|$ for $x_{j} \in \mathscr{T}$. Thus $f_{*}$ and hence $f_{l}(j=1,2)$ are BV-functionals. But since $f_{j}^{*}(x)=f_{j}\left(x^{*}\right)$, we have $f$ in the complex span of $P^{\prime}$. That is $\mathrm{BV}(\mathscr{A}) \subset\left(P^{\prime}-P^{\prime}\right)+$ $i\left(P^{\prime}-P^{\prime}\right)$. Conversely let $f \in \mathbf{C}$-span $(P)$. Then $f=f_{1}+i f_{2}$ where $f_{l}$ is BV and $f_{,}^{*}(x)=f_{l}\left(x^{*}\right)(j=1,2)$. Therefore $f$ admits a complex representing measure $\mu_{f}$ whose real and imaginary parts are $\mu_{f_{1}}$ and $\mu_{f_{2}}$ respectively. It now follows from Remark 2.2.4 that $f$ is $\mathrm{BV}$ and the proof is complete. 
Corollary to Theorem 2.2. A linear functional $f$ is $\mathrm{BV}$ if and only if

$$
\|f\|_{0}=\lim _{(\mathcal{J}, n)} \sum_{\sigma}\left(\begin{array}{l}
n \\
i_{1}
\end{array}\right) \cdots\left(\begin{array}{l}
n \\
i_{k}
\end{array}\right) \mid f\left(\Pi x_{\imath}^{\sigma_{i}}\left(1-x_{\imath}^{1-\sigma_{i}}\right) \mid<\infty,\right.
$$

where $\sigma$ is a 0-1 function on the first $k$ natural numbers and the limit is taken over all finite subsets $\left\{x_{1}, \cdots, x_{k}\right\}$ of $\mathscr{T}$ and all integers $n$. Moreover $\|f\|_{0}=\|f\|$.

Proof. The collection $\mathscr{P}=\{x, 1-x \mid x \in \mathscr{T}\}$ of simple cycles is an admissible collection of cycles as mentioned above.

3. Application to classical moment problems. The classical moment problem is one of characterizing functions $f$ which admit integral representations of the form

$\left(^{*}\right) \quad f\left(n_{1}, \cdots, n_{\nu}\right)=\int_{K} t_{1}^{n_{1}} \cdots t_{\nu}^{n_{\nu}} d \mu(t)$,

where $K$ is a subset of $\nu$-dimensional space $\mathbf{R}^{\nu}$. Restrictions are put on $\mu$. Here we apply the results of $\$ 2$ to solve this moment problem for compact convex sets $K$ with nonvoid interior and with respect to both nonnegative and signed $\mu$.

To put this in our setting let $\mathscr{A}(x)$ be the algebra of all polynomials in the $\nu$-variables $x=x_{1} \cdots x_{\nu}$. A subset $\mathscr{T}$ of polynomials, nonnegative on $K$ such that

(i) $\mathscr{A}(x)=\operatorname{Alg} \operatorname{span} \mathscr{T}$

(ii) $1-\boldsymbol{p} \in A \operatorname{Alg} \operatorname{span}^{+}(\mathscr{T})$ whenever $\boldsymbol{p} \in \mathscr{T}$

(iii) $K=\bigcap_{p \in T}\left\{t \in \mathbf{R}^{\nu} \mid \boldsymbol{p}(t) \geqq 0\right\}$

can be constructed.

3.1. Construction of $\mathscr{T}$. Let $t^{0}$ be an interior point of $K$ and $B(K)=\left\{\boldsymbol{p}\right.$-affine $\mid \boldsymbol{p}(t) \geqq 0$ for all $t \in K$ and $\left.\boldsymbol{p}\left(t^{0}\right)=1\right\}$.

LEMMA 3.1.1. $B(K)$ is uniformly bounded.

Proof. There exists $\epsilon>0$ such that the Euclidean disk $S_{\epsilon}\left(t^{0}\right)=$ $\left\{t \in \mathbf{R}^{\nu} \mid\left\|t-t^{0}\right\|<\epsilon\right\}$ is contained in $K$. If $p \in B(K)$ assumes its supremum on $K$ at $t^{1}\left(\sup \boldsymbol{p}(K)=\boldsymbol{p}\left(t^{1}\right)\right)$ then $t^{1}$ is an extreme point of $K$ and thus $t^{1} \notin S_{\epsilon}\left(t^{0}\right)$. Also there exists $t^{2} \notin S_{\epsilon}\left(t^{0}\right)$ such that $t^{0}=$ $\alpha t^{1}+(1-\alpha) t^{2} \quad(0<\alpha<1)$. Therefore $\epsilon<\left\|t^{2}-t^{0}\right\|=\alpha\left\|t^{1}-t^{2}\right\|<\alpha$. $(\operatorname{diam}(K))$ so $\alpha>\epsilon / \operatorname{diam}(K)$ where $\operatorname{diam} K=\sup \{\|s-t\| \mid s, t \in K\}$. Since $1=\alpha p\left(t^{1}\right)+(1-\alpha) p\left(t^{2}\right)$, we have 


$$
\boldsymbol{p}\left(t^{1}\right)=\frac{1-(1-\alpha) \boldsymbol{p}\left(t^{2}\right)}{\alpha} \leqq \frac{1}{\alpha}<(\operatorname{diam}(K)) / \epsilon
$$

LeMma 3.1.2. $B(K)$ is a compact base for $P_{A}=\{\boldsymbol{p}$-affine $\mid \boldsymbol{p} \geqq 0$ on $K\}$.

Proof. If we norm the finite dimensional space $A(K)$ of affine functions on $K$ by $\|\boldsymbol{p}\|_{K}=\sup |\boldsymbol{p}(K)|$. Lemma 3.1.1 implies $B(K)$ is compact the evaluation functional $\hat{t}^{0}$ defined by $\hat{t}^{0}(\boldsymbol{p})=\boldsymbol{p}\left(t^{0}\right)$ is strictly positive on $P_{A}$ and the assertion follows since $B(K)$ is contained in the preimage of 1 under $\hat{t}^{0}$.

Proposition 3.1.3. The set $\mathscr{T}=\left\{\boldsymbol{p} /\|\boldsymbol{p}\|_{K} \mid \boldsymbol{p} \in \operatorname{ext} B(K)\right\} \cup\{0\}$ of normalized extreme points of $B(K)$ along with 0 satisfies 3.(i), 3.(ii) and 3.(iii).

Proof. Since $A(K)$ is finite dimensional, and $B(K)$ is convex and compact, $B(K)=\operatorname{co}[\operatorname{ext}(B(K))]$. Therefore, $\operatorname{span}^{+} \mathscr{T}=P_{A}$ and since $[\boldsymbol{p}-\inf \boldsymbol{p}(K)] /[\sup \boldsymbol{p}(K)-\inf \boldsymbol{p}(K)] \in P_{A}$ for every $\boldsymbol{p} \in A(K)$ we have span $\mathscr{T}=A(K)$ so that (i) follows. (ii) is clear since $0 \leqq \boldsymbol{p} \leqq 1$ for all $p \in \mathscr{T}$ and $\operatorname{span}^{+} \mathscr{T}=P_{A}$. The Hahn-Banach Theorem implies $K=\bigcap_{p \in P_{A}}\{t \mid \boldsymbol{p}(t) \geqq 0\}$ and since $P_{A}=\operatorname{span}^{+} \mathscr{T}$ we have $\bigcap_{p \in \mathscr{T}}\{t \mid \boldsymbol{p}(t) \geqq 0\}$ is also $K$ and (iii) follows.

In the event that $K$ is a convex polytope, the set $\mathscr{T}$ of Proposition 3.1.3 can be more precisely described as the linear functionals which expose the faces of $K$. To be explicit there exist a minimal set of linear functionals $\quad\left\{L_{l} \mid i=1,2, \cdots, k\right\} \quad$ such that $K=\bigcap_{1}\left\{t \mid L_{l}(t) \geqq\right.$ $\left.\inf L_{l}(K)\right\}$. Let $\boldsymbol{p}_{l}(K)=\left[L_{l}(t)-\inf L_{l}(K)\right] /\left[\sup L_{l}(K)-\inf L_{l}(K)\right]$.

Proposition 3.1.4. $\mathscr{T}=\left\{\boldsymbol{p}_{l} \mid j=1, \cdots, k\right\} \cup\{1,0\}$.

Proof. Let $\boldsymbol{p} \in \operatorname{ext} B(K)$ and $Z(\boldsymbol{p})=\{t \in K \mid \boldsymbol{p}(t)=0\}$. If $Z(\boldsymbol{p})=$ $\varnothing$ then $\inf \boldsymbol{p}(K)>0$ so $\boldsymbol{p}$ is no smaller (with respect to the ordering induced by $P_{A}$ ) than the constant function whose value is $\inf \boldsymbol{p}(K)$. Lemma 3.1.2 implies $\boldsymbol{p}$ is a multiple of this function so that $\boldsymbol{p} \equiv 1$. If $Z(\boldsymbol{p}) \neq \varnothing$ then $Z(\boldsymbol{p})$ is a subset of a maximal proper extreme convex subset (face) of $K$. But the faces of $K$ are precisely the sets $Z\left(\boldsymbol{p}_{l}\right)$ cf. [9]. Since $Z(\boldsymbol{p})$ is extremal we have ext $Z(\boldsymbol{p}) \subset \operatorname{ext} Z\left(\boldsymbol{p}_{i}\right) \subset$ $\operatorname{ext}(K)$. But $\operatorname{ext}(K)$ is finite. Hence there exists $\alpha>0$ such that $\boldsymbol{p}-\alpha \boldsymbol{p}_{i}$ is nonnegative on $\operatorname{ext}(K)$, whence $\boldsymbol{p}-\alpha \boldsymbol{p}_{j} \in P_{A}$ so that $\boldsymbol{p}=\alpha \boldsymbol{p}_{\imath}$ or $\mathscr{T} \subset\left\{\boldsymbol{p}_{3}\right\}$. Conversely if $\boldsymbol{p} \in P_{A}$ and $\boldsymbol{p}_{3}-\boldsymbol{p} \in P_{A}$ then $Z\left(\boldsymbol{p}_{3}\right) \subset$ $Z(\boldsymbol{p})$. But $Z\left(\boldsymbol{p}_{\jmath}\right)$ is a face and hence is a maximal proper extremal convex subset of $K$. If $\boldsymbol{p} \neq \equiv 1$ then $Z(\boldsymbol{p})$ is a proper extremal convex subset of $K$ so that $Z(\boldsymbol{p})$ is exactly the face $Z\left(\boldsymbol{p}_{l}\right)$. But every face of a 
convex polytope affinely spans a hyperplane $H$, c.f. [9]. In as much as $\boldsymbol{p}$ and $\boldsymbol{p}_{j}$ agree on $H$ we must have $\alpha \boldsymbol{p}_{j}=\boldsymbol{p}$ or $\boldsymbol{p}_{j}$ is on an extreme ray of $\boldsymbol{P}_{\boldsymbol{A}}$. Thus $\mathscr{T} \subset\left\{\boldsymbol{p}_{j}\right\}$.

3.2. Solution of the moment problem. Let $\mathscr{T}$ be any subset of $\mathscr{A}(x)$ which satisfies 3.(i), 3.(ii) and 3.(iii).

Proposition 3.2.1. A multiplicative linear functional $\chi$ is $\mathscr{T}$ positive if and only if there exist $t \in K$ such that $\chi(\boldsymbol{p})=\boldsymbol{p}(t)$. Moreover the set of positive multiplicative linear functionals $\Gamma$ on $\mathscr{A}(x)$ is homeomorphic to $K$ under the map $\chi \rightarrow t$.

Proof. Let $\mathbf{p}_{i}$ be the polynomial defined by $\boldsymbol{p}_{i}(x)=x_{\imath}$ (evaluation at the $i$ th coordinate) and let $t_{i}=\chi\left(\boldsymbol{p}_{i}\right)$. If $\boldsymbol{p} \in \mathscr{T}$ such that $\boldsymbol{p}(x)=$ $\sum \alpha_{i_{1}, \cdots, l_{\nu}} x_{1}^{t_{1}} \cdots x_{\nu}^{t_{\nu}}$ then $\chi(\boldsymbol{p})=\sum \alpha_{i_{1}, \cdots, t_{\nu}} t_{1}^{i_{1}} \cdots t_{\nu}^{t_{\nu}}$, so $\chi(p)=p\left(t_{1} \cdots t_{\nu}\right)$ for all $p \in \tau$ and hence all $p$. The rest of the assertion follows easily.

A linear functional $L$ in $\mathscr{A}(x)$ is uniquely determined by its moments $f\left(n_{1}, \cdots, n_{\nu}\right)=L\left(t_{1}^{n_{1}} \cdots t_{\nu}^{n_{\nu}}\right)$.

Corollary 3.2.2. A real valued function $f$ on the $\nu$-tuples of nonnegative integers admits a real integral representation of the form

$$
f\left(n_{1}, \cdots, n_{\nu}\right)=\int_{K} t_{1}^{n_{1}} \cdots t_{\nu}^{n_{\nu}} d \mu_{f}
$$

if and only if the linear functional $L_{f}$ determined by the moments $f\left(n_{1}, \cdots, n_{\nu}\right)$ is $\mathscr{T}-\mathrm{BV} ; \mu_{f}$ being nonnegative whenever $L_{f}$ is $\mathcal{T}$-positive.

In order to more precisely describe those functions which are solutions to the moment problem let $E_{\imath}$ denote the shift operator as defined by $\left(E_{\imath} f\right)\left(n_{1}, \cdots, n_{\nu}\right)=f\left(n_{1}, \cdots, n_{i}+1, \cdots, n_{\nu}\right)$ for each $i=$ $1,2, \cdots, \nu$. We define the product of two shift operators by composition and let $\mathscr{A}(E)$ denote the algebraic span of the shift operators. Then $\mathscr{A}(E)$ is isomorphic to $\mathscr{A}(x) ; p\left(E_{1}, \cdots, E_{\nu}\right)$ being mapped onto $p\left(x_{1}, \cdots, x_{\nu}\right)$.

THEOREM 3.2.2. A function $f$ is a solution to the classical moment problem $\left({ }^{*}\right)$ with respect to a positive measure $\mu$ if and only if

$$
\left[\boldsymbol{p}_{1} \cdots \boldsymbol{p}_{k}(E)\right] f(0) \geqq 0 \text { for all } \boldsymbol{p}_{1}, \cdots, \boldsymbol{p}_{k} \in \mathcal{T} \quad(0=(0, \cdots, 0)) ;
$$

and $f$ is a solution with respect to a signed measure if and only if

$$
\lim \sum\left(\begin{array}{c}
n \\
i_{1}, \cdots
\end{array}\right) \cdots\left(\begin{array}{c}
n \\
i_{1 k}, \cdots
\end{array}\right)\left|\left[\Pi_{l, p} \boldsymbol{p}_{l_{l}}(E)\right] f(0)\right|<\infty
$$


where the limit is taken over all finite subsets of a set of cycles which include each $\boldsymbol{p} \in \mathcal{T}$.

Proof. Let $\boldsymbol{p}(x)=\sum \alpha_{n_{1}, \cdots, n_{\nu}} x_{1}^{n_{1}} \cdots x_{\nu}^{n_{\nu}}$. Then

$$
\begin{aligned}
L_{f}(\boldsymbol{p}) & =\sum \alpha_{n_{1}, \cdots, n_{\nu}} f\left(n_{1}, \cdots, n_{\nu}\right) \\
& =\sum \alpha_{n_{1}, \cdots, n_{\nu}} E_{1}^{n_{1}} \cdots E_{\nu}^{n_{\nu}} f(0) \\
& =[\boldsymbol{p}(E) f](0) .
\end{aligned}
$$

Thus $L_{f}\left(\boldsymbol{p}_{1} \cdots \boldsymbol{p}_{k}\right)=\left(\left[\left(\boldsymbol{p}_{1} \cdots \boldsymbol{p}_{k}\right)(E)\right] f\right)(0)$ and the assertion follows.

Corollary 3.2.3. The set $P=A \operatorname{Ag} \operatorname{span}^{+}(\mathscr{T})$ is uniformly dense in the nonnegative continuous functions on $K$.

Proof. Let $f$ be a continuous function which is nonnegative on $K$ but not in the uniform closure of $P$. The Hahn-Banach theorem implies the existence of a linear functional $L$ on $C(K)$ such that $L(f)<0 \leqq$ $\inf L(P)$. But then $L$ admits a nonnegative representing measure $\mu_{L}$ on $K$ such that $L(\boldsymbol{p})=\int_{K} \boldsymbol{p} d \mu_{L}$ for all $\boldsymbol{p} \in \mathscr{A}$. Since $f$ is nonnegative on $K$ we reach the contradiction that $L(f)=\int_{K} f d \mu_{L} \geqq 0$.

\subsection{Specific examples}

\subsubsection{The Hausdorfi (1-dimensional) moment} problem. Take $K=[a, b]$, then from Proposition 3.1.4,

$$
\mathscr{T}=\left\{1,0,\left(\frac{1}{b-a} x-\frac{a}{b-a}\right)\right\}
$$

so that if $(E f)(n)=f(n+1)$, then Theorem 3.2.2 implies:

$f$ is a solution of the moment problem $\int_{0}^{1} t^{n} d \mu=f(n)$ if and only if

(i) $\lim _{n} \frac{1}{(b-a)^{n}} \sum_{i=0}^{n}\left(\begin{array}{c}n \\ i\end{array}\right)\left|(E-a I)^{i}(-E+b I)^{n-i} F(a)\right|<\infty$.

Moreover $\mu$ is nonnegative if and only if

(ii) $(E-a I)^{i}(-E+b I)^{j} f(0) \geqq 0$

for all pairs on nonnegative integers $i$ and $j$. 
Note that if $a=0$ and $b=1$ then $-E+b$ is the difference operator $\Delta \equiv I-E$ and (i) reduces to $\lim _{n} \sum\left(\begin{array}{l}n \\ i\end{array}\right)\left|\Delta^{n-i} f(i)\right|<\infty$ while (ii) becomes $\Delta^{\prime} f(i) \geqq 0 ;$ Hausdorff's well known solution to the little moment problem, c.f. [6 or 14]. In this case Corollary 3.2 .3 implies that every continuous function on $[0,1]$ can be uniformly approximate by polynomials of the form $\sum \alpha_{i j} t^{\prime}(1-t)^{\prime},\left(\alpha_{i j}>0\right)$, an obvious corollary to the theory of Bernstein polynomials.

3.3.2. The 3-dimensional cube. $K=\left\{t \in R^{3} \mid 0 \leqq t_{1} \leqq 1\right.$, $i=1,2,3\}$. Then $\mathscr{T}=\left\{1,0,1-x_{1}, 1-x_{2}, 1-x_{3}, x_{1}, x_{2}, x_{3}\right\}$ so that $f$ is a solution of $\left(^{*}\right)$ if and only if

$$
\lim _{n} \sum\left(\begin{array}{l}
n \\
i_{1}
\end{array}\right)\left(\begin{array}{l}
n \\
i_{2}
\end{array}\right)\left(\begin{array}{l}
n \\
i_{3}
\end{array}\right)\left|\left(I-E_{1}\right)^{n-t_{1}}\left(I-E_{2}\right)^{n-z_{2}}\left(I-E_{3}\right)^{n-i_{2}} f\left(i_{1}, i_{2}, i_{3}\right)\right|<\infty
$$

and $\mu$ is nonnegative if and only if $\left(I-E_{1}\right)^{i_{1}}\left(I-E_{2}\right)^{2}\left(I-E_{3}\right)^{i_{3}} f\left(j_{1}, j_{2}, j_{3}\right) \geqq$ 0 for all choices of nonnegative integers, $i_{1}, i_{2}, i_{3}, j_{1}, j_{2}, j_{3}$.

3.3.3. The 2-dimensional simplex. If $K=\left\{t \mid 1-t_{1}-t_{2} \geqq\right.$ $\left.0, t_{1} \geqq 0, t_{2} \geqq 0\right\}$, then $\mathscr{T}=\left\{1,0,1-x_{1}-x_{2}, x_{1}, x_{2}\right\}$. Thus $f$ is a solution of $\left(^{*}\right)$ if and only if

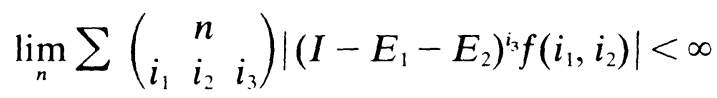

and the measure $\mu$ of determinancy is nonnegative if and only if $\left(I-E_{1}-E_{2}\right)^{t_{3}} f\left(i_{1}, i_{2}\right) \geqq 0$ for all choices of nonnegative integers $i_{1}, i_{2}$ and $i_{3}$. Corollary 3.2.3 implies that every continuous function on $K$ can be uniformly approximated by polynomials of the form

$$
\sum \alpha_{111_{213}} t_{1}^{t_{1}^{\prime}} t_{2}^{l_{2}}\left(1-t_{1}-t_{2}\right)^{l_{3}} \quad\left(\alpha_{111_{213}}>0\right) \text {; }
$$

a fact which also follows from [8, page 51.]

4. Applications to semigroups. Let $S$ be a commutative semigroup with identity 1 and involution ${ }^{*}$ c.f.[8]. For each $x \in X$ we define the shift operator $E_{x}$ on the functions on $S$ by $E_{x} f(y)=$ $f(x y)$. The product $E_{x} E_{y}$ of two shift operators is defined by composition and the linear sum is defined by $\left(\alpha E_{x}+\beta E_{y}\right) f(z)=$ $\alpha f(x z)+\beta f(y z)$. Note that $E_{x} E_{y}=E_{x y}$ for all $x, y \in S$. The algebra $\mathscr{A}(E)$ spanned by the shift operators is then a commutative algebra with identity $E_{1}$ which can be made into an involution algebra by defining $\left(\sum \alpha_{i} E_{x_{i}}\right)^{*}=\Sigma \alpha_{1}^{*} E_{x_{i}}$. The functions on $S$ can be biuniquely identified 
with the linear functionals $L_{f}$ on $\mathscr{A}(E)$ by $L_{f}\left(\sum \alpha_{1} E_{x_{i}}\right)=\sum \alpha_{i} f\left(x_{i}\right)$. Then the multiplicative functionals are homeomorphic to the semigroup semicharacters relative to the topology of simple convergence. Thus the $\mathscr{T}$-positive and $\mathrm{BV}$-functionals on $\mathscr{A}(E)$ correspond to certain classes of functions on $S$. Various possibilities exist for $\mathscr{T}$. In defining $\mathscr{T}$ more generality is obtained by considering a generator set for $S$, i.e. a subset $X$ of $S$ such that every element of $\mathscr{T}$ is a product of finitely many elements of $X \cup X^{*}$.

4.1. Completely monotonic functions. We assume $x^{*}=$ $x$ for all $x \in S$ and set $\mathscr{T}=\left\{E_{x}, 1-E_{x} x \in X\right\}$. Then $L_{f}$ is positive (or $\mathrm{BV}$ ) if and only if $f$ is completely monotonic cf. [5] (or BV in the sense of [11]). If $S=(\{0,1, \cdots\},+)$, then these are the solutions to the little moment problem described in 3.3.1. If $S$ is the set of all triples of nonnegative integers under coordinate addition, the situation is as described in $\$ 3.3 .2$.

4.2. Moment problems in $\mathbf{R}^{\nu}$. For a given convex body in $\mathbf{R}^{\nu}$ we let $S$ be the semigroups of all $\nu$-tuples of nonnegative integers under coordinate addition and the identity involution. We define $\mathscr{T}$ as in Theorem 3.2.2. Then the $\mathscr{T}$-BV functionals can be viewed as those functions which are solutions of the moment problem of $\$ 3 .\left({ }^{*}\right)$. The situation is clarified by the examples given in $\$ 3$.

\subsection{Positive definite functions on groups and involu-} tion semigroups. If $S$ is a group or inverse semigroup (c.f. [2]), then $x^{*}=x^{-1}$ provides a natural involution on $S$. A natural involution on the semigroup of all order pairs of nonnegative integers which is considered in [10] is given by $(i, j)^{*}=(j, i)$. For arbitrary involution semigroup with generator set $X$ we define a difference operator $T_{\sigma_{\mathrm{x}}}=$ $1 / 2 I+(\sigma / 4) E_{x}+\left(\sigma^{*} / 4\right) E_{x^{*}}$, for each $x \in S$ and fourth root of unit $\sigma . \mathscr{T}$ is then selected as $\left\{T_{\sigma_{x}} \mid x \in X\right\}$. A linear functional $L_{f}$ on $\mathscr{A}(E)$ is $\mathcal{T}$-positive if and only if finite products of the form $\left(\Pi, T_{\sigma, x} f\right)(1)$ are nonnegative for all fourth roots of unity $\sigma$, and all choices of $x, \in X$. Such functions $f$ on $S$ are called (X)-positive definite. Moreover $L_{f}$ is $\mathscr{T}$-BV if and only if

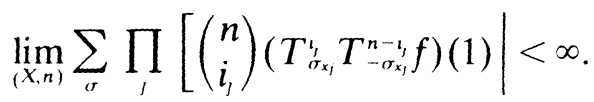

The functions $f$ on $S$ for which $L_{f}$ is $\mathscr{T}$-BV are called $X$-BV functions on $S$. These functions are completely discussed in [12] and the results of $\$ 2$ can be used to recover the theorems there. When $S$ is a group, $\Gamma$ 
corresponds to the group characters $\operatorname{Char}(S)$ and Theorem 2.1 takes the form of Bochner theorem for discrete groups.

COROLlaRy 4.3.1. If $S$ is a commutative group, then a complex valued function $f$ is positive definite if and only if $f$ admits an integral representation

$$
f(x)=\int_{\text {Chars }} \chi(x) d \mu(\chi) .
$$

In particular the classically defined positive definite functions on groups agree with those defined here. Theorem 2.2 describes those functions on $S$ which are in the span of the positive definite functions.

When $S$ is the semigroup of pairs of nonnegative integers as described above and $X=\{(1,0),(1,1)\}$ then the multiplicative linear functional $L_{\chi} \in \Gamma$ can be identified with the points of the unit disk $D$ and Theorems 2.1 and 2.2 characterize those functions $f$ on $S$ which are solutions of the moment problem $f(i, j)=\int_{D} z^{i}\left(z^{*}\right)^{j} d \mu(z)$ for nonnegative and complex $\mu$ respectively. The solution to this moment problem for nonnegative $\mu$ leads to a derivation of the spectral theorem for normal operators [10]. For general involution semigroups, if we select $X=S$ then $\Gamma$ corresponds to the bounded semicharacters $\chi$ such that $\chi\left(x^{*}\right)=\chi(x)$ and Theorem 2.1 shows that $f$ is $\mathscr{T}$-positive if and only if $f$ is ${ }^{*}$-definite as defined in [7].

5. Applications to $B *$-algebras. Let $\mathscr{A}$ be a commutative $B^{*}$-algebra with identity 1 such that $\|x\|=\left\|x^{*}\right\|$ and let $\mathscr{T}=\left\{x x^{*} \mid\|x\|<\right.$ 1\}. Then each $x \in \mathscr{A}$ can be expressed as $x_{1}+i x_{2}$ where $x_{j}^{*}=x$, $(j=1,2)$. Thus

$$
\alpha x=[(\alpha-1)+(\alpha-1) i] 1+\left[1-\alpha\left(1-x_{1}\right)\right]+\left[1-\alpha\left(1-x_{2}\right)\right] i .
$$

If we choose $\alpha>0$ such that $\left\|\alpha\left(1-x_{l}\right)\right\|<1(j=1,2)$ then the square root lemma implies the existence of $y_{l} \in \mathscr{T}$ such that $y_{l}=1-\alpha\left(1-x_{l}\right)$ or

$$
\alpha x=[(\alpha-1)+(\alpha-1) i] 1+y_{1}+y_{2} .
$$

Thus $\mathscr{T}$ generates $\mathscr{A}$. The square root lemma also implies $1-x \in \mathscr{T}$ whenever $x \in \mathscr{T}$. If we let $\Gamma$ be the set of multiplicative linear functionals $\chi$ such that $\chi^{*}(x)=\chi\left(x^{*}\right)$, then Theorems 2.1 and 2.2 establish the following. 
$f(x)=\int_{\Gamma} \chi(x) d \mu(x)$ with respect to a necessarily unique regular Borel measure $\mu$ if and only if

$$
\lim _{(\mathscr{T}, n)} \sum\left(\begin{array}{c}
n \\
i_{1}
\end{array}\right) \cdots\left(\begin{array}{c}
n \\
i_{k}
\end{array}\right)\left|f\left[\prod_{j} x_{j}^{\prime,}\left(1-x_{j}\right)^{n-t_{1}}\right]\right|<\infty .
$$

Also $f$ admits a nonnegative representing measure if and only if $f\left(x x^{*}\right) \geqq 0$ for all $x \in \mathscr{A}$.

The second assertion is well known, c.f. [15].

\section{REFERENCES}

1. H. Bauer, Konvexität in Topologischen Vektorräumen, Lecture Notes University of Hamburg, Hamburg, West Germany.

2. A. H. Clifford and G. B. Preston, The Algebraic Theory of Semigroups (I), Amer. Math. Soc., Providence (1967).

3. N. Dunford and J. Schwartz, Linear Operators (I), New York, 1958.

4. G. Choquet, Lectures on Analysis (I), (II) and (III), New York/Amsterdam, 1969.

5. N. Fine and P. H. Maserick, On the simplex of completely monotonic functions on a commutative semigroup, Can. J. Math., (1970), 317-326.

6. J. Kist and P. H. Maserick, BV-functions on semilattices, Pacific J. Math., (1971), 711-723.

7. R. J. Lindahl and P. H. Maserick, Positive-definite functions on involution semigroups, Duke Math. J., (1971), 771-782.

8. G. G. Lorentz, Bernstein Polynomials, Toronto, 1953.

9. P. H. Maserick, Convex polytopes in linear spaces, Illinois J. Math., (1965), 623-635.

10. Spectral theory of operator-valued transformations, J. of Math. Anal. and applications, (1973), 497-507.

11. - Moment and BV-functions on commutative semigroups, Trans. Amer. Math. Soc., 181 (1973), 61-75.

12. - $B V$-functions, positive definite functions and moment problems, to appear in the Trans. Amer. Math. Soc.

13. M. A. Naimark, Normed Rings, The Netherlands, 1970.

14. S. E. Newman, Measure algebras and functions of bound variation on idempotent semigroups, Trans. Amer. Math. Soc., 163 (1972), 189-205.

15. R. R. Phelps, Lectures on Choquet's Theorems, Princeton, 1966.

16. D. V. Widder, The Laplace Transform, Princeton, 1946.

Received October 7, 1975.

The Pennsylvania State University

UNIVERSITY PARK, PA 16802 



\section{Pacific Journal of Mathematics}

Vol. 68, No. 1

March, 1977

Richard Julian Bagby, On $L^{p}, L^{q}$ multipliers of Fourier transforms . .......

Robert Beauwens and Jean-Jacques Van Binnebeek, Convergence theorems in

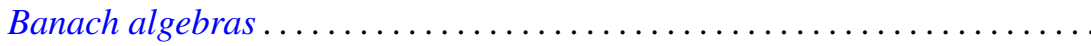

James Cyril Becker, Skew linear vector fields on spheres in the stable

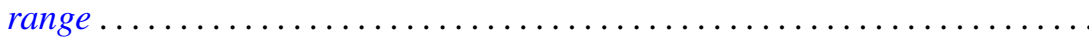

Michael James Beeson, Continuity and comprehension in intuitionistic formal

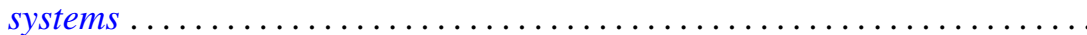

James K. Deveney, Generalized primitive elements for transcendental field

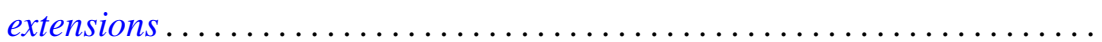

Samuel S. Feder, Samuel Carlos Gitler and K. Y. Lam, Composition properties

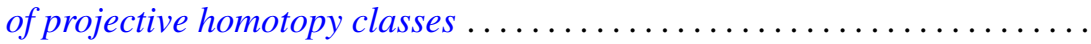

Nathan Jacob Fine, Tensor products of function rings under composition ......

Benno Fuchssteiner, Iterations and fixpoints . . . . . . . . . . . . . .

Wolfgang H. Heil, On punctured balls in manifolds

Shigeru Itoh, A random fixed point theorem for a multivalued contraction

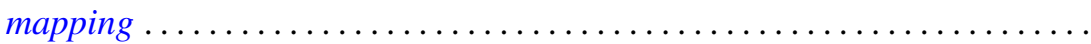

Nicolas P. Jewell, Continuity of module and higher derivations . . . . . . ......

Roger Dale Konyndyk, Residually central wreath products . . . . . . . . . . .

Linda M. Lesniak and John A. Roberts, On Ramsey theory and graphical

parameters.

Vo Thanh Liem, Some cellular subsets of the spheres.

Dieter Lutz, A perturbation theorem for spectral operators

P. H. Maserick, Moments of measures on convex bodies ... . . .

Stephen Joseph McAdam, Unmixed 2-dimensional local domains . .

D. B. McAlister and Norman R. Reilly, E-unitary covers for inverse semigroups...

William H. Meeks, III and Julie Patrusky, Representing codimension-one

homology classes by embedded submanifolds . . .

Premalata Mohapatro, Generalised quasi-Nörlund summability . .

Takahiko Nakazi, Superalgebras of weak-*Dirichlet algebras .

Catherine Louise Olsen, Norms of compact perturbations of operators .

William Henry Ruckle, Absolutely divergent series and isomorphism of

subspaces. II.

Bernard Russo, On the Hausdorff-Young theorem for integral operators .

Arthur Argyle Sagle and J. R. Schumi, Anti-commutative algebras and

homogeneous spaces with multiplications ............

Robert Evert Stong, Stiefel-Whitney classes of manifolds .

D. Suryanarayana, On a theorem of Apostol concerning Möbius functions of

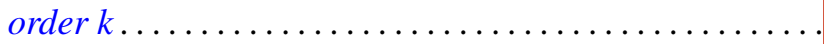

Yoshio Tanaka, On closedness of $C$ - and $C^{*}$-embeddings . . 